\title{
A new characterization of projective special linear groups $L_{3}(q)$
}

\section{B. Ebrahimzadeh}

\author{
Communicated by A. Yu. Olshanskii
}

\begin{abstract}
A BSTRACT. In this paper, we prove that projective special linear groups $L_{3}(q)$, where $0<q=5 k \pm 2(k \in \mathbb{Z})$ and $q^{2}+q+1$ is a prime number can be uniquely determined by their order and the number of elements with same order.
\end{abstract}

\section{Introduction}

Let $G$ be a finite group, $\pi(G)$ be the set of prime divisors of the order of $G$ and $\pi_{e}(G)$ be the set of the order of elements in $G$. If $k \in \pi_{e}(G)$, then we denote the number of elements of order $k$ in $G$ by $m_{k}(G)$ and the set of the numbers of elements with the same order in $G$ by nse $(G)$. In other words, nse $(G)=\left\{m_{k}(G): k \in \pi_{e}(G)\right\}$. Also we denote a Sylow $p$-subgroup of $G$ by $G_{p}$ and the number of Sylow $p$-subgroups of $G$ by $n_{p}(G)$. The prime graph $\Gamma(G)$ of group $G$ is a graph whose vertex set is $\pi(G)$, and two vertices $u$ and $v$ are adjacent if and only if $u v \in \pi_{e}(G)$. Moreover, assume that $\Gamma(G)$ has $t(G)$ connected components $\pi_{i}$, for $i=1,2, \ldots, t(G)$. In the case where $G$ is of even order, we assume that $2 \in \pi_{1}$.

The characterization of groups by nse $(G)$ pertains to Thompson's problem (Problem 12.37 in [10]) which Shi posed in [13]. The first time, this type of characterization was studied by Shao and Shi. In [12], they proved that if $S$ is a simple $K_{4}$ - group,then $S$ is characterizable by nse $(S)$ and $|S|$. Following this result, in $[2-4,7,8,11]$, it is proved that sporadic

2020 MSC: 20D06, 20D60.

Key words and phrases: element orders, the number of elements with same order, prime graph, projective special linear group. 
simple groups, projective special linear groups $L_{2}(p)$ and suzuki groups $S z(q)$, where $q-1$ is a prime number and also Ree groups ${ }^{2} G_{2}(q)$, where $q \pm \sqrt{3 q}+1$ is a prime number can be uniquely determined by their orders and $\operatorname{nse}(G)$.

In this paper, we prove that projective special linear groups $L_{3}(q)$, where $0<q=5 k \pm 2(k \in \mathbb{Z})$ and $q^{2}+q+1$ is a prime number can be uniquely determined by their orders and the number of elements with same order of the group. In fact, we prove the following main theorem.

Main Theorem. Let $G$ be a group with $|G|=\left|L_{3}(q)\right|$ and nse $(G)=$ nse $\left(L_{3}(q)\right)$, where $0<q=5 k \pm 2(k \in \mathbb{Z})$ and $q^{2}+q+1$ is a prime number. Then $G \cong L_{3}(q)$.

\section{Notation and preliminaries}

Lemma 2.1 ([6]). Let $G$ be a Frobenius group of even order with kernel $K$ and complement $H$. Then

(a) $t(G)=2, \pi(H)$ and $\pi(K)$ are vertex sets of the connected components of $\Gamma(G)$;

(b) $|H|$ divides $|K|-1$;

(c) $K$ is nilpotent.

Definition 2.2. A group $G$ is called a 2-Frobenius group if there is a normal series $1 \unlhd H \unlhd K \unlhd G$ such that $G / H$ and $K$ are Frobenius groups with kernels $K / H$ and $H$ respectively.

Lemma 2.3 ([1]). Let $G$ be a 2-Frobenius group of even order. Then

(a) $t(G)=2, \pi(H) \cup \pi(G / K)=\pi_{1}$ and $\pi(K / H)=\pi_{2}$;

(b) $G / K$ and $K / H$ are cyclic groups satisfying $|G / K|$ divides $|\operatorname{Aut}(K / H)|$.

Lemma $2.4([15])$. Let $G$ be a finite group with $t(G) \geqslant 2$. Then one of the following statements holds:

(a) $G$ is a Frobenius group;

(b) $G$ is a 2-Frobenius group;

(c) $G$ has a normal series $1 \unlhd H \unlhd K \unlhd G$ such that $H$ and $G / K$ are $\pi_{1}$-groups, $K / H$ is a non-abelian simple group, $H$ is a nilpotent group and $|G / K|$ divides $\mid$ Out $(K / H) \mid$.

Lemma $2.5([5])$. Let $G$ be a finite group and $m$ be a positive integer dividing $|G|$. If $L_{m}(G)=\left\{g \in G \mid g^{m}=1\right\}$, then $m|| L_{m}(G) \mid$. 
Lemma 2.6. Let $G$ be a finite group. Then for every $i \in \pi_{e}(G), \varphi(i)$ divides $m_{i}(G)$, and $i$ divides $\sum_{j \mid i} m_{j}(G)$. Moreover, if $i>2$, then $m_{i}(G)$ is even.

Proof. By Lemma 2.5, the proof is straightforward.

Lemma 2.7 ([14]). Let $G$ be a non-abelian simple group such that $(5,|G|)=1$. Then $G$ is isomorphic to one of the following groups:

(a) $L_{n}(q), n=2,3, q \equiv \pm 2(\bmod 5)$;

(b) $G_{2}(q), q \equiv \pm 2(\bmod 5)$;

(c) ${ }^{2} A_{2}(q), q \equiv \pm 2(\bmod 5)$;

(d) ${ }^{3} D_{4}(q), q \equiv \pm 2(\bmod 5)$;

(e) ${ }^{2} G_{2}(q), q=3^{2 m+1}, m \geqslant 1$.

Lemma 2.8. Let $L$ be the projective special linear groups $L_{3}(q)$, where $p=q^{2}+q+1$ is a prime number. Then $m_{p}(L)=(p-1)|L| /(3 p)$ and for every $i \in \pi_{e}(L)-\{1, p\}, p$ divides $m_{i}(L)$.

Proof. First we know that $\left|L_{3}(q)\right|=q^{3}\left(q^{3}-1\right)\left(q^{2}-1\right)$. Now since $\left|L_{p}\right|=p$, it follows that $L_{p}$ is a cyclic group of order $p$. Thus $m_{p}(L)=\varphi(p) n_{p}(L)=$ $(p-1) n_{p}(L)$.Now it is enough to show $n_{p}(L)=|L| /(3 p)$. By the result in[15] that yields that the prime $p$ is an isolated vertex of $\Gamma(L)$.Hence $\left|C_{L}\left(L_{p}\right)\right|=p$ and $\left|N_{L}\left(L_{p}\right)\right|=x p$ for a natural number $x$. We know that $N_{L}\left(L_{p}\right) / C_{L}\left(L_{p}\right)$ embed in $\operatorname{Aut}\left(L_{p}\right)$, which implies $x \mid p-1$.Furthermore, by Sylow's Theorem, $n_{p}(L)=\left|L: N_{L}\left(L_{p}\right)\right|$ and $n_{p}(L) \equiv 1(\bmod p)$. Therefore $p$ divides $|L| /(x p)-1$.Thus $q^{2}+q+1$ divides $\left(q^{3}\left(q^{3}-1\right)\left(q^{2}-\right.\right.$ 1)/ $(x p)-1$.It follows that $q^{2}+q+1$ divides $q^{6}-q^{5}-q^{4}+q^{3}-x$ so we have $p \mid 3-x$ and since $x \mid p-1$, we deduce that $x=3$, and the proof is finished. Let $r \in \pi_{e}(L)-\{1, p\}$. Since $p$ is an isolated vertex of $\Gamma(L)$, it follows that $p \nmid r$ and $p r \notin \pi_{e}(L)$. Thus $L_{p}$ acts fixed point freely on the set of elements of order $r$ by conjugation and hence $\left|L_{p}\right| \mid m_{r}(L)$. So we conclude that $p \mid m_{r}(L)$.

\section{Proof of the main theorem}

In this section, we prove the main theorem in the following lemmas. We denote the projective special linear groups $L_{3}(q)$, by $L$ and prime number $q^{2}+q+1$ by $p$. Recall that $G$ is a group with $|G|=|L|$ and $\operatorname{nse}(G)=\operatorname{nse}(L)$.

Lemma 3.1. $m_{2}(G)=m_{2}(L), m_{p}(G)=m_{p}(L), n_{p}(G)=n_{p}(L), p$ is an isolated vertex of $\Gamma(G)$ and $p \mid m_{k}(G)$ for every $k \in \pi_{e}(G)-\{1, p\}$. 
Proof. By Lemma 2.6 if $\mathrm{r}=2$ then $m_{2}(G)=\phi(2) n_{2}(G)=|G| / 6=|L| / 6=$ $m_{2}(L)$.Thus it follows that $m_{2}(G)=m_{2}(L)$.According to Lemma 2.6, $\left(m_{p}(G), p\right)=1$. Thus $p \nmid m_{p}(G)$ and hence Lemma 2.8 implies that $m_{p}(G) \in\left\{m_{1}(L), m_{2}(L), m_{p}(L)\right\}$. Moreover, $m_{p}(G)$ is even, so we deduce that $m_{p}(G)=m_{p}(L)$. Since $G_{p}$ and $L_{p}$ are cyclic groups of order $p$ and $m_{p}(G)=m_{p}(L)$, we deduce that $m_{p}(G)=\varphi(p) n_{p}(G)=\varphi(p) n_{p}(L)=$ $m_{p}(L)$, so $n_{p}(G)=n_{p}(L)$.

Now we prove that $p$ is an isolated vertex of $\Gamma(\mathrm{G})$. Assume the contrary. Then there is $t \in \pi(G)-\{p\}$ such that $t p \in \pi_{e}(G)$. So $\mathrm{m}_{t p}(G)=$ $\varphi(t p) n_{p}(G) k$, where $k$ is the number of cyclic subgroups of order $t$ in $C_{G}\left(G_{p}\right)$. Since $n_{p}(G)=n_{p}(L)$, it follows that $m_{t p}(G)=(t-1)(p-$ 1) $|L| k /(3 p)$. If $m_{t p}(G)=m_{p}(L)$, then $t=2$ and $k=1$. Furthermore, Lemma 2.6 since $p>2$ so $m_{p}(G)$ is even. The other hand $p \mid 1+m_{p}(G)$. Now by Lemma $2.5 p \mid m_{p}(G)$ so $p \mid 1+m_{2}(G)$. Since $m_{p}(G)=m_{2 p}(G)$ so $p \mid 1+m_{2 p}(G)$ yields $p \mid m_{2}(G)+m_{2 p}(G)$. Since $m_{2}(G)=m_{2}(L)$ and $p \mid m_{2}(L)$, we deduce that $p \mid m_{2 p}(G)$ which is a contradiction. So Lemma 2.8 implies that $p \mid \mathrm{m}_{t p}(G)$. Hence $p \mid t-1$ and since $m_{t p}(G)<|G|$, we deduce that $p-1 \leqslant 3$. But this is impossible because $p=q^{2}+q+1$.

Let $k \in \pi_{e}(G)-\{1, p\}$. Since $p$ is an isolate vertex of $\Gamma(G), p \nmid k$ and $p k \notin \pi_{e}(G)$.Thus $G_{p}$ acts fixed point freely on the set of elements of order $k$ by conjugation and hence $\left|G_{p}\right| \mid m_{k}(G)$. So we conclude that $p \mid m_{k}(G)$.

Lemma 3.2. The group $G$ is neither a Frobenius group nor a 2-Frobenius group.

Proof. Let $G$ be a Frobenius group with kernel $K$ and complement $H$. Then by Lemma 2.1, $t(G)=2$ and $\pi(H)$ and $\pi(K)$ are vertex sets of the connected components of $\Gamma(G)$ and $|H|$ divides $|K|-1$. Now by Lemma 3.1, $p$ is an isolate vertex of $\Gamma(G)$. It follows that (i) $|H|=p$ and $|K|=|G| / p$ or (ii) $|H|=|G| / p$ and $|K|=p$. Since $|H|$ divides $|K|-1$, we deduce that the case (i) can not occur. So $|H|=p$ and $|K|=|G| / p$, hence $q^{2}+q+1 \mid \frac{q^{3}\left(q^{3}-1\right)\left(q^{2}-1\right)}{q^{2}+q+1}-1$, so we have $q^{2}+q+1 \mid \frac{\left(q^{8}-q^{6}-q^{5}-q^{3}\right)}{q^{2}+q+1}-1$ in the way we conclude $\left.q^{2}+q+1 \mid\left(q^{2}+q+1\right)\left(q^{4}-2 q^{3}+3 q-3\right)+2\right)$. Thus $p \mid 2$ which is impossible.

We now show that $G$ is not a 2-Frobenius group. Let $G$ be a 2Frobenius group. Then $G$ has a normal series $1 \unlhd H \unlhd K \unlhd G$ such that $G / H$ and $K$ are Frobenius groups by kernels $K / H$ and $H$ respectively. Set $|G / K|=x$. Since $p$ is an isolated vertex of $\Gamma(G)$, we have $|K / H|=p$ and $|H|=|G| /(x p)$. By Lemma 2.3, $|G / K|$ divides $|\operatorname{Aut}(K / H)|$. Thus 
$x \mid p-1$ and since $\left(q^{2}+q, q^{2}+q+1\right)=1$, now we deduce $q^{2}+q+1|H|$. Therefore $H_{t} \rtimes K / H$ is a Frobenius group with kernel $H_{t}$ and complement $K / H$, where $t=q^{2}+q+1$. So $|K / H|$ divides $\left|H_{t}\right|-1$. It implies that $q^{2}+q+1 \mid\left(q^{2}+q+1\right)-1$, but this is a contradiction.

Lemma 3.3. The group $G$ is isomorphic to the group $L$.

Proof. By Lemma 3.1, $p$ is an isolated vertex of $\Gamma(G)$. Thus $t(G)>1$ and $G$ satisfies one of the cases of Lemma 2.4. Now Lemma 3.2 implies that $G$ is neither a Frobenius group nor a 2-Frobenius group. Thus only the case (c) of Lemma 2.4 occurs. So $G$ has a normal series $1 \unlhd H \unlhd K \unlhd G$ such that $H$ and $G / K$ are $\pi_{1}$-groups, $K / H$ is a non-abelian simple group. Since $p$ is an isolated vertex of $\Gamma(G)$, we have $p|| K / H \mid$. On the other hand, we know that $5 \nmid|G|$.Thus $K / H$ is isomorphic to one of the groups in Lemma 2.7. Hence we consider the following isomorphisms.

(1) If $K / H \cong L_{2}\left(q^{\prime}\right)$, where $q^{\prime} \equiv \pm 2(\bmod 5)$, then by(table Id) [15], $\pi\left(L_{2}\left(q^{\prime}\right)=q^{\prime},\left(q^{\prime} \pm 1\right) / d\right.$, where $d=\left(2, q^{\prime}-1\right)$. We assume $d=1$, so $p^{\prime}=$ $q^{\prime} \pm 1$. Now we consider $q^{2}+q+1=q^{\prime} \pm 1$. The first case if $q^{2}+q+1=q^{\prime}+1$ then $q(q+1)=q^{\prime}$, that is a contradiction, because $q^{\prime}=p^{\prime m}$. The second case if $q^{2}+q+1=q^{\prime}-1$ then we deduce $q^{2}+q+2=q^{\prime}$,now since $\left|L_{2}\left(q^{\prime}\right)\right| \nmid|G|$, hence we have a contradiction. Now if $d=2$ then $p^{\prime}=q^{\prime},\left(q^{\prime} \pm 1\right) / 2$ so $q^{2}+q+1=q^{\prime}$, since $\left|L_{2}\left(q^{\prime}\right)\right| \nmid|G|$, so we have a contradiction. In the way if $q^{2}+q+1=\left(q^{\prime}+1\right) / 2$ then $2 q^{2}+2 q+1=q^{\prime}$ since $\left|L_{2}\left(q^{\prime}\right)\right| \nmid|G|$, hence we have a contradiction. For other case if $q^{2}+q+1=\left(q^{\prime}-1\right) / 2$ then $2 q^{2}+2 q+3=q^{\prime}$. But $\left|L_{2}\left(q^{\prime}\right)\right| \nmid|G|$, so we have a contradiction.

(2) If $K / H \cong G_{2}\left(q^{\prime}\right)$, where $q^{\prime} \equiv \pm 2(\bmod 5)$, then by(table Ic) [15], $\pi\left(G_{2}\left(q^{\prime}\right)=q^{\prime 2} \pm q^{\prime}+1\right.$. Thus if $q^{2}+q+1=q^{\prime 2}+q^{\prime}+1$ then $q(q+1)=q^{\prime}\left(q^{\prime}+1\right)$ in conclude $q=q^{\prime}$. The other hand we know that $|K / H|$ must be divided $|G|$, but we can see easily that $q^{6}\left(q^{6}-1\right)\left(q^{2}-1\right) \nmid q^{3}\left(q^{3}-1\right)\left(q^{2}-1\right)$, hence we have a contradiction. Now we consider $q^{2}+q+1=q^{\prime 2}-q^{\prime}+1$, since $\left(q^{\prime}, q^{\prime}-1\right)=1$ we deduce $q^{\prime}=q+1$. The other hand we know $\left|G_{2}\left(q^{\prime}\right)\right|$ must be divided $|G|$. But we can see easily that $\left.\left.(q+1)^{6}(q+1)^{6}-1\right)(q+1)^{2}-1\right) \nmid$ $q^{3}\left(q^{3}-1\right)\left(q^{2}-1\right)$ that is a contradiction.

(3) If $K / H \cong U_{3}\left(q^{\prime}\right)$, where $q^{\prime} \equiv \pm 2(\bmod 5)$, then by(table Ic) [15], $\pi\left(U_{3}\left(q^{\prime}\right)\right)=\left(q^{\prime 3}-1\right) /\left(q^{\prime}+1\right)\left(3, q^{\prime}+1\right)$. First if $\left(3, q^{\prime}+1\right)=1$, then we consider $q^{2}+q+1=q^{\prime 2}-q^{\prime}+1$, hence $q(q+1)=q^{\prime}\left(q^{\prime}-1\right)$, in conclude $q^{\prime}=q+1$. We know that $\left|U_{3}\left(q^{\prime}\right)\right|$ must be divided $|G|$, but we can see easily that $\left.\left.(q+1)^{3}(q+1)^{3}+1\right)(q+1)^{2}-1\right) \nmid q^{3}\left(q^{3}-1\right)\left(q^{2}-1\right)$ that is a contradiction. For the other case we have a contradiction, similarily.

(4) If $K / H \cong{ }^{2} G_{2}\left(q^{\prime}\right)$, where $q^{\prime}=3^{2 m+1}$, then by(table Id) [15], $\pi\left({ }^{2} G_{2}\left(q^{\prime}\right)\right)=q^{\prime} \pm \sqrt{3 q^{\prime}}+1$. First we consider $q^{2}+q+1=q^{\prime} \pm \sqrt{3 q^{\prime}}+1$, 
in conclude $3^{m+1}\left(3^{m} \pm 1\right)=q(q+1)$, since $(q, q+1)=1$ so $q+1=3^{m+1}$, $q=3^{m}+1$. In conclude $3^{m+1}\left(3^{m}+1\right)=\left(3^{m+1}-1\right)\left(3^{m+1}\right)$ then we have $3^{m}+1=3^{m+1}-1$ that is a contradiction. Now if $q^{2}+q+1=q^{\prime}-\sqrt{3 q^{\prime}}+1$ then $q(q+1)=3^{m+1}\left(3^{m}-1\right)$, since $(q, q+1)=1$ hence $q=3^{m}-1$ and $q+1=3^{m+1}$, first if $q=3^{m}-1$, then by attention to the above equation we have $3^{m}-1\left(3^{m}\right)=3^{m+1}\left(3^{m}-1\right)$, that in finally we deduce $3^{m}=3^{m+1}$, that this a contradiction. Now for the other case if $q+1=3^{m+1}$, then $3^{m+1}\left(3^{m+1}-1\right)=3^{m+1}\left(3^{m}-1\right)$, that we deduce $3^{m+1}-1=3^{m}-1$. That is a contradiction.

(5) If $K / H \cong{ }^{3} D_{4}\left(q^{\prime}\right)$ then by(table Ic) [15], $\pi\left({ }^{3} D_{4}\left(q^{\prime}\right)=q^{4}-q^{2}+1\right.$. Now we consider $q^{2}+q+1=q^{\prime 4}-q^{\prime 2}+1$ in conclude $q^{\prime 2}\left(q^{\prime 2}-1\right)=q(q+1)$, by attention to $(q, q+1)=1$ so $q=q^{\prime 2}-1$. Now since $\left.\right|^{3} D_{4}\left(q^{\prime}\right)|\nmid| G \mid$ that is a contradiction.

Hence we deduce that $K / H \cong L_{3}\left(q^{\prime}\right)$. As a result $|K / H|=L_{3}\left(q^{\prime}\right)$. Since $p$ is an isolated vertex and also $p|| K / H \mid$, we consider $q^{2}+q+1=$ $q^{\prime 2}+q^{\prime}+1$. As aresult $q=q^{\prime}$ and also since $1 \unlhd H \unlhd K \unlhd G$, we deduce that $H=1, G=K \cong L$.

\section{References}

[1] G. Y. Chen, About Frobenius groups and 2-Frobenius groups, J. Southwest China Normal University, 20, no. 5, 1995, 485-487.

[2] B. Ebrahimzadeh, R. Mohammadyari, A new characterization of symplectic group $C_{2}\left(3^{n}\right)$, Acta et commentationes universitatis tartuensis de mathematica, 23, no. 1 , (2019).

[3] B. Ebrahimzadeh, A. Iranmanesh, H. Parvizi Mosaed, A new characterization of Ree group ${ }^{2} G_{2}(q)$ by the order of group and number of elements with same order, Int. J. Group Theory, 6, no. 4, 2017, pp. 1-6.

[4] B. Ebrahimzadeh, R. Mohammadyari, A new characterization of suzuki groups, Archivum mathematicum(Brono) Tomus, 55(2019), 17-21.

[5] G. Frobenius, Verallgemeinerung des sylow'schen satzes, Berliner sitz, 1895, 981983.

[6] D. Gorenstein, Finite groups, Harper and Row, New York, 1980.

[7] A. Khalili Asboei, A. Iranmanesh, Characterization of the linear groups $L_{2}(p)$, Czechoslovak Mathematical Journal, 64, no. 139, 2014, 459-464.

[8] A. Khalili Asboei, S. S. Amiri, A. Iranmanesh, A. Tehranian, A new characterization of sporadic simple groups by NSE and order, J. Algebra Appl., 12, no.2, 2013, 1250158 .

[9] A. S. Kondrat'ev, Prime graph components of finite simple groups, Mathematics of the USSR-Sbornik, 67, no. 1, 1990, 235-247.

[10] V. D. Mazurov, E. I. Khukhro, eds., Unsolved problems in group theory, The Kourovka Notebook, 16 ed. Inst. Mat. Sibirsk. Otdel. Akad. Novosibirsk (2006). 
[11] H. Parvizi Mosaed, A. Iranmanesh, A. Tehranian, Characterization of suzuki group by nse and order of group, Bull. Korean Math. Soc., 53, no. 3, 2016, 651-656.

[12] C. G. Shao, W. Shi, Q. H. Jiang, Characterization of simple $K_{4}$-groups, Front Math China, 3, no. 3, 2008, 355-370.

[13] W. J. Shi, A new characterization of the Sporadic simple groups, J. Group Theory, Proc. of the 1987 Singapore Conf., Walter de Gruyter, Berlin, 1989, 531-540.

[14] W. J. Shi, A characterization of $U_{3}\left(2^{n}\right)$ by their element orders J. Southwest-China Normal Univ, 25, no. 4, 2000, 353-360.

[15] J.S. Williams, Prime graph components of finite groups, J. Algebra, 69, no. 2, 1981, 487-513.

[16] A.V. Zavarnitsine, Recognition of the simple groups $L_{3}(q)$ by element orders, $J$. Group Theory, 7, no. 1, 2004, 81-97.

\section{CONTACT INFORMATION}

Behnam

Ebrahimzadeh

University of Applied Science and Technology (UAST), ITMC Center, Shiraz, Iran E-Mail(s): behnam.ebrahimzadeh@gmail.com

Received by the editors: 16.08 .2018

and in final form 09.05.2020. 